\title{
Differential Cerebrovascular Toxicity of Various Tobacco Products: A Regulatory Perspective
}

\section{Ravi K Sajja, Pooja Naik and Luca Cucullo*}

Department of Pharmaceutical Sciences, Texas Tech University Health Sciences Center, Amarillo, USA

*Corresponding author: Luca Cucullo, Department of Pharmaceutical Sciences, Texas Tech University Health Sciences Center, Amarillo, USA, Tel: +1-806-43-1000; Email: luca.cucullo@ttuhsc.edu

Rec date: Jan 02, 2015; Acc date: Jan 02, 2015; Pub date: Jan 09, 2015

Copyright: (C) 2015 Sajja RK. This is an open-access article distributed under the terms of the Creative Commons Attribution License, which permits unrestricted use, distribution, and reproduction in any medium, provided the original author and source are credited.

\section{Editorial}

Blood-Brain Barrier (BBB) is a dynamic anatomical interface that separates brain parenchyma from blood circulation and is principally constituted by the cerebral microcapillary endothelial cells $[1,2]$. BBB is composed of distinct structural and functional organization through the presence of inter-endothelial tight junction complexes, abundant expression of nutrient and efflux transporters including metabolically active sites. While the TJ complexes tightly seal the paracellular gaps and contribute to high resistance of BBB [3], the presence of specific nutrient transporters and receptor systems selectively regulate the delivery of metabolic substrates, nutrients and macromolecules to the brain. In addition, efflux transporters belonging to the $A B C$ superfamily prevent the brain permeation of blood-borne neurotoxic chemicals including xenobiotics and eliminates the accumulation of toxic metabolites within the brain parenchyma [1]. Taken together, the $\mathrm{BBB}$ serves as a physiological, transport and metabolic barrier that critically regulates ion, molecular and cellular flux into the brain, thus maintaining the CNS microenvironment for optimal neuronal function [2]. Importantly, impairment of BBB integrity by various exogenous or endogenous pathological stimuli involving increased load of oxidative/inflammatory stress in the neurovascular unit, is a potential mechanism underlying the pathogenesis of a host of neurologic and degenerative disorders $[3,4]$.

Tobacco smoking is a serious public health concern and a leading cause of increased rates of preventable mortality and morbidity across the globe with a significant toll on health care costs and personal life ( $1 \%$ of deaths world-wide annually). Cigarette smoke contains a complex mixture of $>8000$ chemicals in vapor and particulate phases such as reactive aldehydes and pro-oxidants with high potential for cellular oxidative injury. Tobacco Smoke (TS) is a potential risk factor in the pathogenesis of various systemic disorders such as cardiovascular diseases, atherosclerosis, insulin resistance and other vascular abnormalities [5]. Mounting evidence suggests that mainstream TS elicits a strong inflammatory and immune response [6], leading to potential endothelial dysfunction and microvascular damage [5]. In addition, both active and passive smoking aggravates endothelial cell activation with significant increase in endothelialleukocyte interactions leading to profound monocyte infiltration and local inflammation $[5,7]$.

Emerging evidence also indicts TS (active and passive) as preventable risk factor in the etiology of neurological disorders such as ischemic stroke, silent cerebral infarctions, multiple sclerosis and cerebral aneurysms [8-10]. Although the precise mechanisms implicated in TS induced neuropathogenesis is not completely understood, nicotine-dependent pathways at the cerebrovasculature are believed to partly mediate TS-induced neurotoxicity and stroke
[11]. Importantly, main stream TS was shown to impair BBB dysfunction that was prevented by anti-oxidant supplementation [7], while nicotine exacerbated ischemic stroke and brain edema [12]. Further, nicotine was also shown to alter BBB permeability in vivo [13] and aggravated post-ischemic inflammatory responses [14]. Thus, it is proposed that $\mathrm{BBB}$ damage is a critical cerebrovascular complication of TS-induced oxidative/inflammatory stress, prodromal to pathogenesis of a host of CNS disorders [7,9].

Currently, various tobacco products are being commercially marketed such as 'reduced exposure', 'light' or 'nicotine-free' products including electronic cigarettes with different levels of nicotine, nitrosamines and other toxic chemicals, claiming a reduced health hazard compared to regular brands. However, experimental and clinical evidence assessing the toxicological profiles of these diverse tobacco products at the brain microvasculature is minimally reported to support their safety and availability of such information is deemed to be essential for regulatory bodies to set standards on the tobacco products for improving public health [15]. Recent studies from our laboratory and others have challenged the safety of these reduced or low-exposure products. For example, recently we investigated the toxic impact of mainstream TS (whole) extracts from various tobacco products on $\mathrm{BBB}$ endothelium in vitro [16]. Our data revealed a strong positive correlation between the TS-induced BBB endothelial dysfunction (through increased oxidative stress) and total tar and nitric oxide content of various tobacco products (such as regular full flavor 3R4F, Ultra low nicotine, Ultra-Light 1R5F, and Nicotine-Free). Mainly, smoke extracts from Nicotine-Free and Ultra low nicotine cigarettes were found to be more toxic at BBB endothelium compared to regular products [16].

In summary, rigorous analysis of the safety (or toxicity) profiles of various tobacco products on cerebrovascular function in experimental and clinical studies is critically required to set up regulatory control. In addition, such studies would provide mechanistic insights into the molecular mechanisms underlying tobacco smoke associated BBB neurotoxicity.

\section{References}

1. Abbott NJ (2013) Blood-brain barrier structure and function and the challenges for CNS drug delivery. J Inherit Metab Dis 36: 437-449.

2. Hawkins BT, Davis TP (2005) The blood-brain barrier/neurovascular unit in health and disease. Pharmacol Rev 57: 173-185.

3. Luissint AC, Artus C, Glacial F, Ganeshamoorthy K, Couraud PO (2012) Tight junctions at the blood brain barrier: physiological architecture and disease-associated dysregulation. Fluids Barriers CNS 9: 23.

4. Zlokovic BV (2008) The blood-brain barrier in health and chronic neurodegenerative disorders. Neuron 57: 178-201. 
Citation: Sajja RK, Naik P, Cucullo L (2015) Differential Cerebrovascular Toxicity of Various Tobacco Products: A Regulatory Perspective. J Pharmacovigil 3: e130. doi:10.4172/2329-6887.1000e130

Page 2 of 2

5. Messner B, Bernhard D (2014) Smoking and cardiovascular disease: mechanisms of endothelial dysfunction and early atherogenesis. Arterioscler Thromb Vasc Biol 34: 509-515.

6. Shiels MS, Katki HA, Freedman ND2, Purdue MP, Wentzensen N, et al (2014) Cigarette smoking and variations in systemic immune and inflammation markers. J Natl Cancer Inst 106.

7. Hossain M, Sathe T, Fazio V, Mazzone P, Weksler B, et al. (2009) Tobacco smoke: a critical etiological factor for vascular impairment at the bloodbrain barrier. Brain Res 1287: 192-205.

8. Chalouhi N, Ali MS, Starke RM, Jabbour PM, Tjoumakaris SI, et al. (2012) Cigarette smoke and inflammation: role in cerebral aneurysm formation and rupture. Mediators Inflamm 2012: 271582.

9. Mazzone P, Tierney W, Hossain M, Puvenna V, Janigro D, et al. (2010) Pathophysiological impact of cigarette smoke exposure on the cerebrovascular system with a focus on the blood-brain barrier: expanding the awareness of smoking toxicity in an underappreciated area. Int J Environ Res Public Health 7: 4111-4126.

10. Salzer J, Hallmans G, Nyström M, Stenlund H, Wadell G, et al. (2013) Smoking as a risk factor for multiple sclerosis. Mult Scler 19: 1022-1027.

11. Piao WH, Campagnolo D, Dayao C, Lukas RJ, Wu J, et al. (2009) Nicotine and inflammatory neurological disorders. Acta Pharmacol Sin 30: 715-722.
12. Paulson JR1, Yang T, Selvaraj PK, Mdzinarishvili A, Van der Schyf CJ, et al. (2010) Nicotine exacerbates brain edema during in vitro and in vivo focal ischemic conditions. J Pharmacol Exp Ther 332: 371-379.

13. Hawkins BT, Abbruscato TJ, Egleton RD, Brown RC, Huber JD, et al. (2004) Nicotine increases in vivo blood-brain barrier permeability and alters cerebral microvascular tight junction protein distribution. Brain Res 19: 48-58.

14. Bradford ST, Stamatovic SM, Dondeti RS, Keep RF, Andjelkovic AV (2011) Nicotine aggravates the brain postischemic inflammatory response. Am J Physiol Heart Circ Physiol 300: H1518-1529.

15. FDA (2012) Food and Drug Administration Center for tobacco products, guidance for industry reporting harmful and potentially harmful constituents in tobacco products and tobacco smoke under section 904 (a) (3) of the Federal Food, Drug, and Cosmetic Act.

16. Naik P, Fofaria N, Prasad S, Sajja RK, Weksler B, et al. (2014) Oxidative and pro-inflammatory impact of regular and denicotinized cigarettes on blood brain barrier endothelial cells: is smoking reduced or nicotine-free products really safe? BMC Neurosci 15: 51 . 